\title{
Laparoscopic surgery reduces the incidence of surgical site infections compared to the open approach for colorectal procedures: a meta-analysis
}

\author{
N. Kulkarni ${ }^{1,3}$ (I) T. Arulampalam ${ }^{2,3}$
}

Received: 8 March 2020 / Accepted: 5 July 2020 / Published online: 9 July 2020

(c) Springer Nature Switzerland AG 2020

\begin{abstract}
Background Surgical site infections (SSI) are the commonest healthcare associated infections. They severely compromise patient safety, are a significant burden on healthcare resources and have an adverse impact on patient quality of life. The incidence of SSIs can be as high as $10 \%$ after colorectal procedures. The laparoscopic approach is being increasingly used to undertake colorectal procedures. It provides advantages over the traditional open approach with smaller incisions, shorter hospital stay and equal oncological outcomes. The aim of this meta-analysis was to evaluate whether the laparoscopic approach for colorectal procedures reduces the incidence of SSI compared to the open approach.

Methods Randomised controlled trials (RCTs) comparing the two approaches published since 2000 were included in the review. Revman 5.3 software was used to carry out the review. Data were pooled and the results were shown as risk ratios with $95 \%$ confidence intervals using the fixed effects model.

Results Sixteen RCT's were included in the analysis comprising 5797 patients. These covered a range of colorectal pathologies including colon cancer, rectal cancer, inflammatory bowel disease and familial adenomatous polyposis syndrome. Analysis showed significantly lower wound infection rates (RR: $0.72,95 \%$ confidence interval: $0.60-0.88, p=0.001$ ) and lower abdominal abscess rates (RR: $0.88,95 \%$ CI $0.62-1.27, p=0.51$ ). The combined SSI rate was significantly lower in laparoscopic compared to open surgery (RR: $0.76,95 \%$ CI $0.64-0.90, p=0.001$ ).

Conclusions Laparoscopic colorectal surgery significantly lowers the incidence of SSI compared to open surgery.
\end{abstract}

Keywords Laparoscopy · Open surgery $\cdot$ Surgical site infections $\cdot$ Wound infections $\cdot$ Colorectal surgery $\cdot$ Enhanced recovery

Electronic supplementary material The online version of this article (https://doi.org/10.1007/s10151-020-02293-8) contains supplementary material, which is available to authorized users.

N. Kulkarni

dr_nvk@yahoo.com

1 Department of Surgery, United Lincolnshire Hospitals NHS Trust, Lincoln, UK

2 Department of Surgery, East Suffolk and North Essex NHS Trust, Colchester, UK

3 Faculty of Health, Social Care and Education, Anglia Ruskin University, Chelmsford, UK

\section{Introduction}

Surgical site infections (SSI) are the commonest healthcare associated infections (HCAI). They severely compromise patient safety, are a significant burden on healthcare resources and have an adverse impact on patient quality of life. The incidence of SSI's can be as high as $10 \%$ after colorectal procedures [1]. The first series of laparoscopic colorectal surgery was reported in 1991 [2]. Numerous multicentre randomised controlled trials (RCTs) carried out internationally have now established the efficacy of the laparoscopic approach for colorectal procedures. A laparoscopic approach is being increasingly used to undertake colorectal procedures. It provides advantages over the traditional open approach with smaller incisions, a shorter hospital stay and equal oncological outcomes. Additional benefits of minimally invasive surgery include significant 


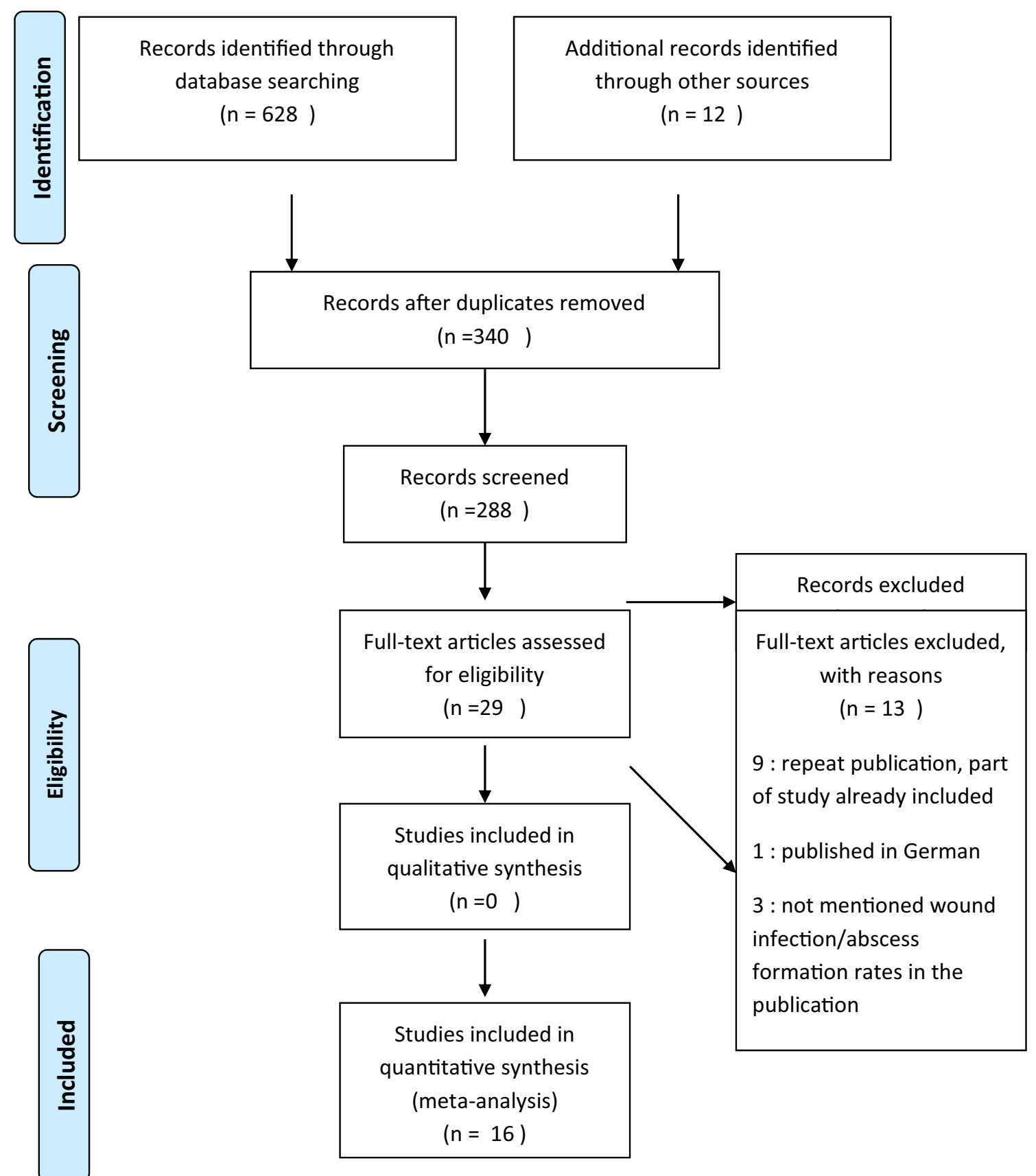

Fig. 1 PRISMA flow diagram

quality improvement in terms of reduced morbidity and suffering for the patient with all the associated inconvenience and cost. There is a significant improvement in the functioning of the health system if these quality improvements lead to sustained reduction in return to hospital and to the operating theatre. These include improved patient flow in hospitals with the capacity created by avoiding unexpected, non-elective returns to hospital, reduced primary care consultations and medication prescriptions and better antibiotic governance. Overall financial cost for the healthcare system can, therefore, be substantially improved. The aim of this study was to evaluate whether the implementation of a laparoscopic approach for colorectal procedures reduces the incidence of SSI compared to the open approach. The studies in the meta-analysis included a range of colorectal pathologies including colon cancer, rectal cancer, Crohn's disease, ulcerative colitis and familial adenomatous polyposis syndrome. 


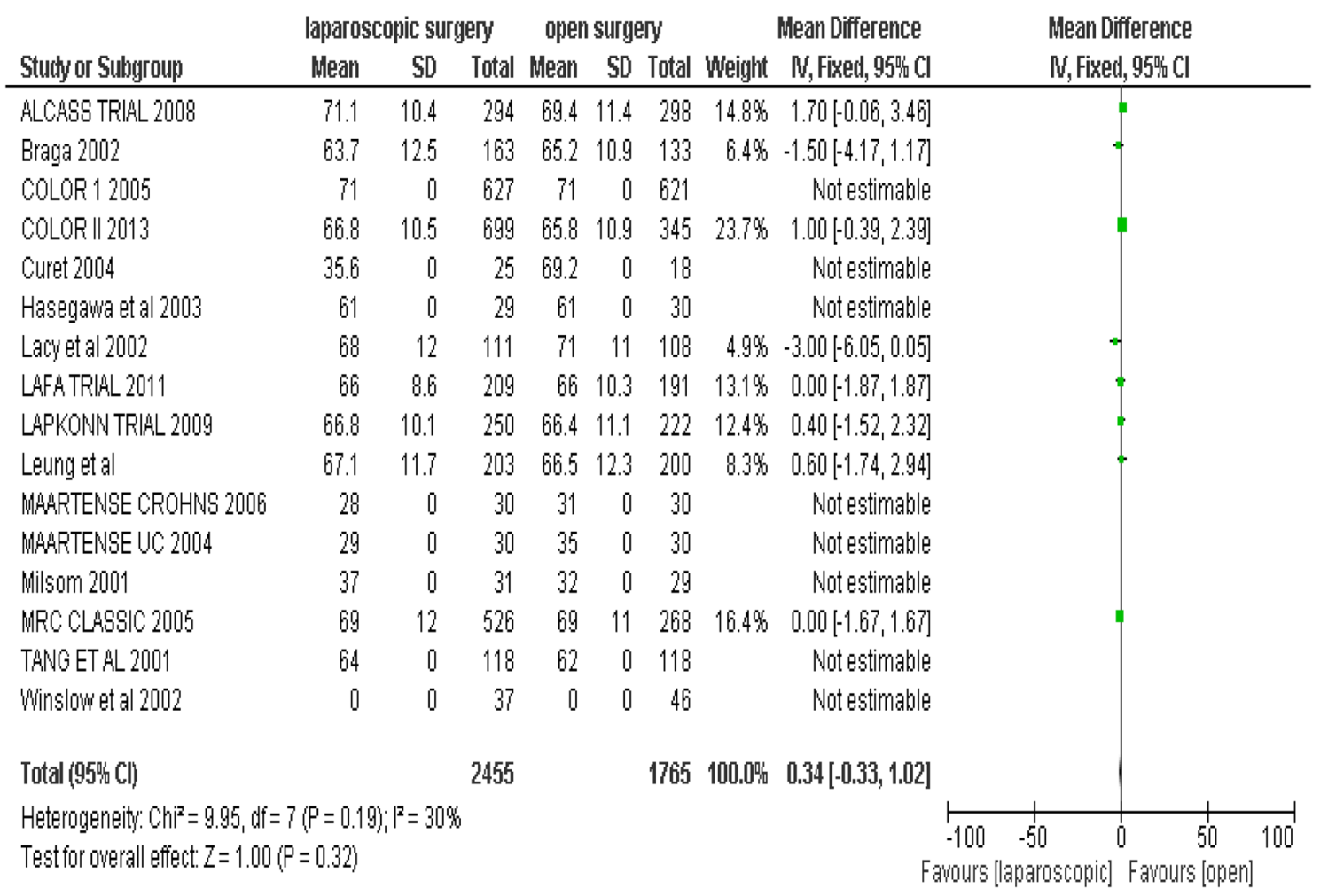

Fig. 2 Age of patients

\section{Materials and methods}

This review and meta-analysis was carried out using validated methodologies [3] and the search strategy outlined below.

\section{Search strategy}

PUBMED, Google scholar, EMBASE, and Cochrane database were searched using the strategy enumerated in the research protocol. Surgical and colorectal journals were searched and society websites (ESCP, ACPGBI, ASGBI, SAGES) were also searched for information about abstracts of presentations in meetings and guidelines. The appropriate medical subject heading terms were used. One researcher reviewed the summary and abstracts (NK). Full text was then reviewed by two reviewers. Data was extracted using a standardised template. Data extracted included type of study, characteristics of the population recruited, pathology included, type of interventions, incidence of wound infections, and risk of bias.

Risk of bias was assessed using the free online Revman 5.3 database. A risk of bias summary figure was also generated.

\section{Inclusion and exclusion criteria}

RCTs published since 2000-2014 comparing outcomes after laparoscopic and open colorectal surgery were included in the study. The studies covered benign and malignant colorectal disease including colon cancer, rectal cancer, Crohn's disease, ulcerative colitis and familial adenomatous polyposis syndrome. Nonrandomised controlled trials and studies published before 2000 were not included in the study.

Sixteen RCT's were included in the meta-analysis. Studies comparing outcomes after laparoscopic and open colorectal surgeries were included. A total of 5797 patients were analysed in total in the studies. Eight of these studies are multicentre [4-11] and 8 were single centre studies [12-19].

The Preferred Reporting Items for Systematic Reviews and Meta-Analyses (PRISMA) flow diagram for our study outlining the process of study selection is shown in Fig. 1. The initial search yielded 628 papers. Twenty-nine full text articles were assessed for eligibility and 16 studies met the criteria for inclusion in the study.

\section{Statistical analysis}

Revman 5.3 software from the Cochrane website was used to compile and present the data in the meta-analysis. The results were depicted by risk ratios with $95 \%$ confidence 


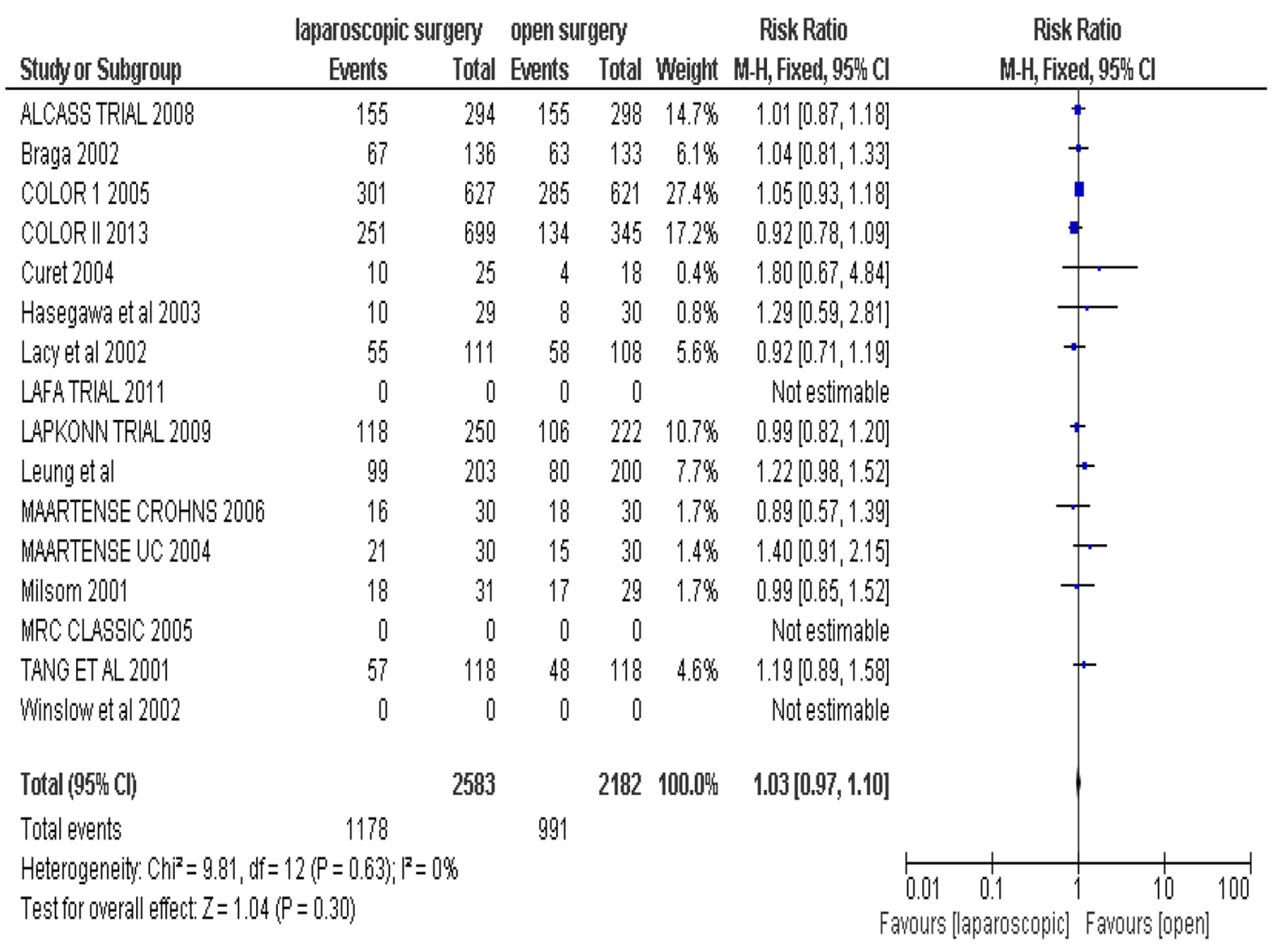

Fig. 3 Female patients

intervals using the fixed effects model. Results were illustrated in forest plots and $p$ value $\leq 0.05$ was considered statistically significant.

\section{Results}

\section{Patient characteristics}

The 16 RCT's included in the study covered a total of 5797 patients. These included 3192 laparoscopic interventions and 2605 open interventions. The interventions were analysed on an intention to treat basis. There was a wide variation in the mean age of the patients amongst the studies. Studies covering malignancies had a higher mean age and studies covering other inflammatory bowel disease had a lower mean age. This goes in line with the general age profile of these patients. However, there was no statistically significant difference in the age groups between laparoscopic and open surgery (see Fig. 2).

Data about the sex of patients was available for 13 studies. Female patients comprised $45.6 \%$ of the total laparoscopic patients (1178/2583) and $45.4 \%$ of the patients undergoing open surgery (991/2182). There was no difference amongst both the groups in terms of sex ratio (see Fig. 3).

\section{Surgical site infections}

All the studies defined SSI in different terms. None of them actually mentioned the abbreviation SSI in their report. However, all the studies covered wound infections. Eight studies also reported abdominal abscess formations as separate from wound infections. Wound infections and abscess rates were assessed separately. Finally, the wound infection and abscess rates were combined to generate a forest plot comparing SSI rates.

Wound infection rates were significantly lower in laparoscopic surgery compared to open surgery (Risk ratio: 0.72 , $95 \%$ CI $0.60-0.88, p=0.001,184$ events in laparoscopic vs 209 in open surgery) (see Fig. 4).

Eight studies reported the abscess formation rates in a total of 3227 patients. Abscess formation rates were also lower in laparoscopic surgery though the $p$ values did not reach significance levels. (Risk ratio: 0.88. 95\% CI $0.62-1.27, p=0.51,69$ events in laparoscopic vs. 51 events in open surgery) (see Fig. 5). 


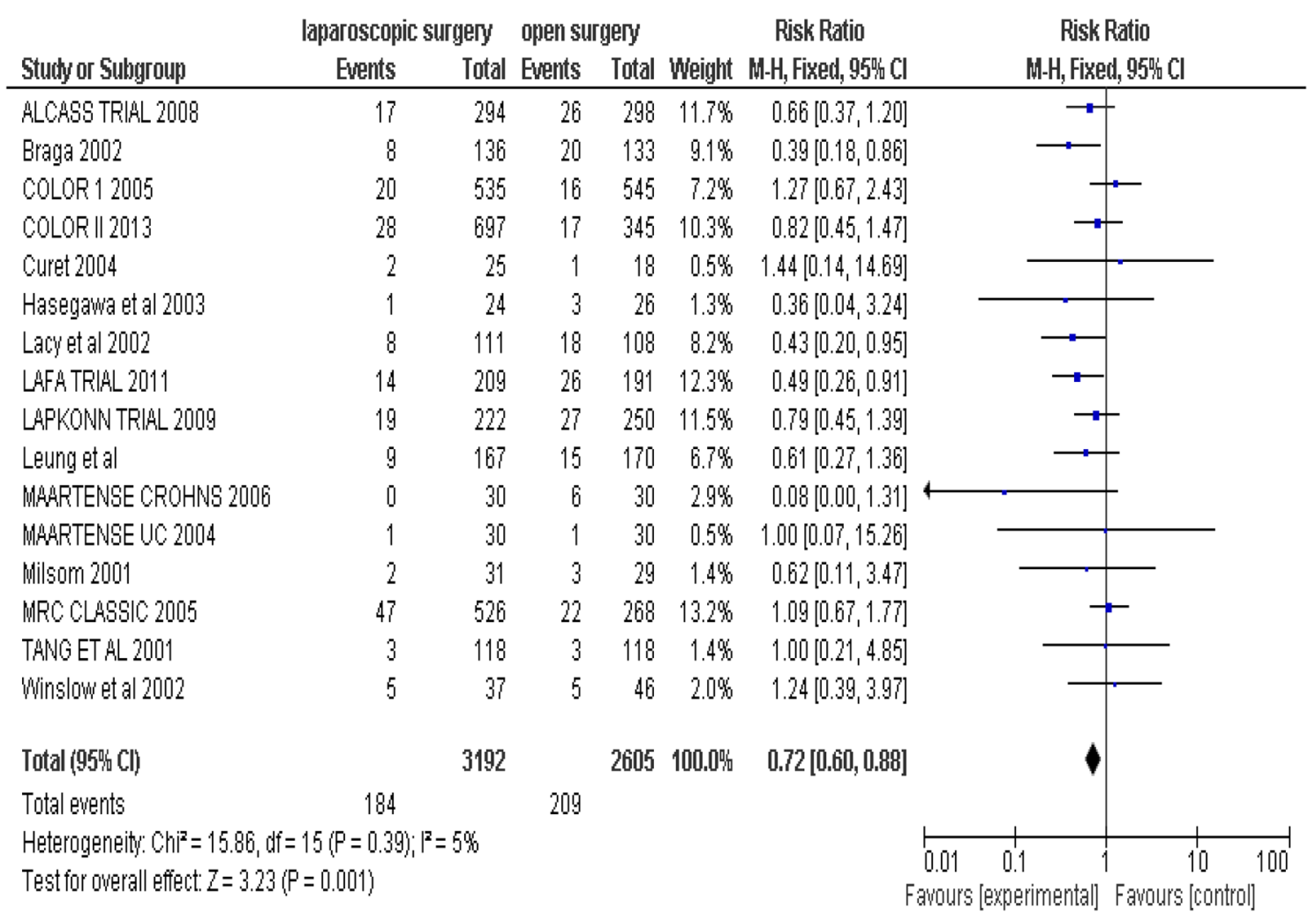

Fig. 4 Forest plot of risk ratio for wound infection for laparoscopic versus open colorectal surgery

Finally the combined SSI rates were significantly lower in the laparoscopic group compared to the open group. (RR: $0.76,95 \%$ CI $0.64-0.90, p=0.001,253$ events in laparoscopic surgery vs 260 events in open surgery) (see Fig. 6).

\section{Discussion}

This study has clearly demonstrated that the incidence of wound infections is significantly lower in laparoscopic compared to open colorectal surgery. SSIs are a major burden on the health system worldwide. They can comprise up to $20 \%$ of all healthcare associated infections [20]. Recent figures have shown that the burden of SSI after colorectal surgery continues to be high (up to 10\%). Public Health England figures have shown that there is a significantly increasing trend in the rate of SSI for patients undergoing large bowel surgery [1]. The studies included in our analysis cover a diverse range of pathologies including colon cancer, rectal cancer, inflammatory bowel disease and familial adenomatous polyposis. Meta-analyses have been carried out comparing short term outcomes after laparoscopic and open colorectal procedures [21-23]. However, these have either included non-randomised controlled trials or not included the whole range of colorectal pathologies as did this study.
None of the previous meta-analyses include studies which have enhanced recovery protocols embedded in perioperative care. One of the RCT's included in our analysis has specifically included enhanced recovery protocols and has concluded that the optimal care for patients undergoing colorectal procedures is through laparoscopic surgery embedded in an enhanced recovery programme [7]. Our results also show that when divided into the different components of superficial and deep space infections, laparoscopic surgery leads to lesser incidence of SSI. Though this is not statistically significant for deep space infections, it goes against the commonly held assumption that laparoscopic surgery does not reduce abscess formation or deep space infections.

The use of laparoscopic surgery for colorectal procedures has steadily increased over the last few years. The National Bowel Cancer Audit Project (NBOCAP) audit, recording management of colorectal cancer in England and Wales, has shown that the number of resections carried out laparoscopically has increased from $44.9 \%$ in 2012-2013 to $61 \%$ in $2017-2018$ [24]. Some units performed up to $80 \%$ of colorectal resections laparoscopically. The conversion rates have remained stable at about $8 \%$. By highlighting the improved SSI rates, our study has shown that this will ultimately be to the benefit of the patients and healthcare resources. The impact has far reaching patient experience, hospital workflow and healthcare economic implications. 


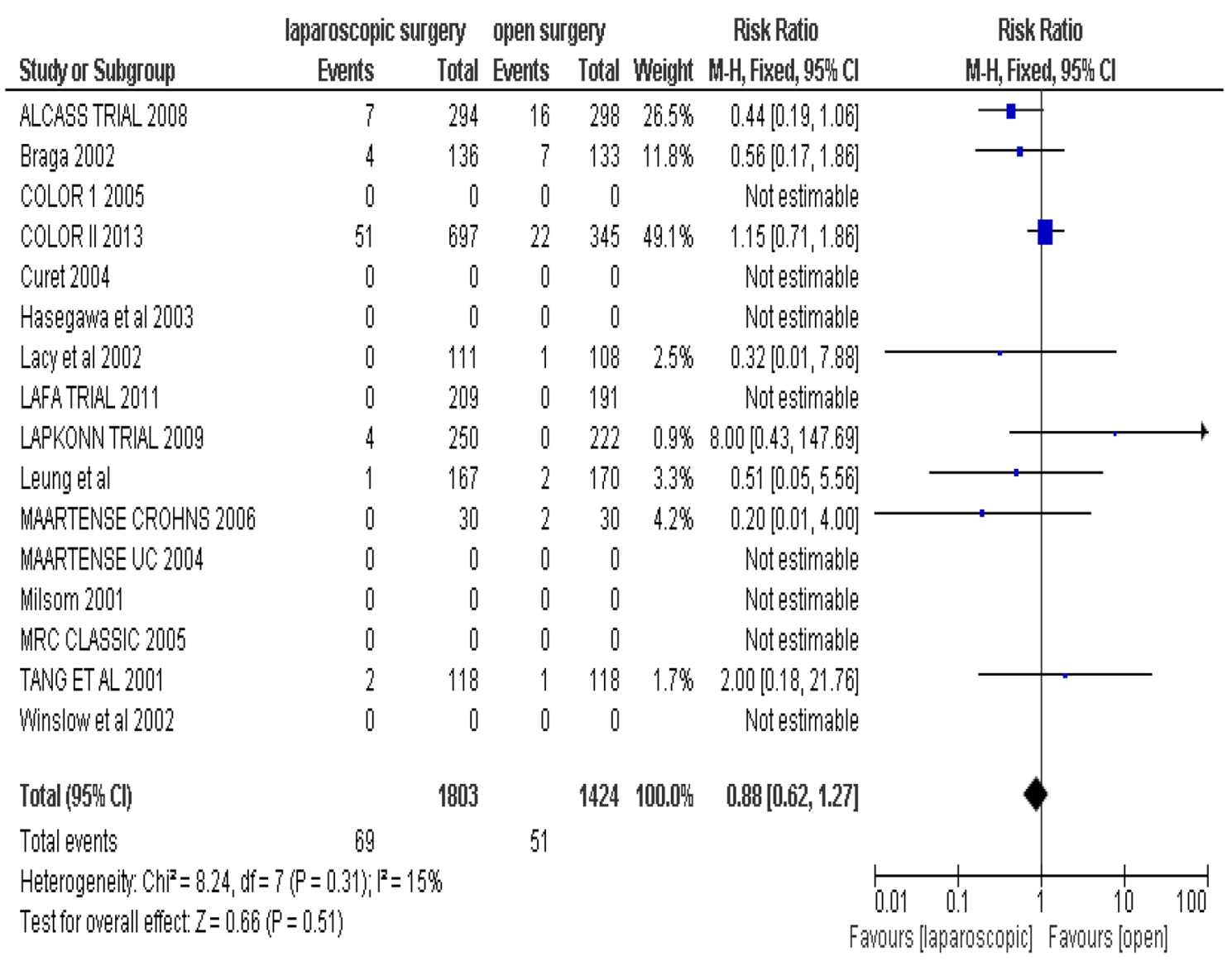

Fig. 5 Forest plot of risk ratio for abdominal abscess formation for laparoscopic versus open colorectal surgery

Investment in training in this technique and capital infrastructure is likely to be offset by the cost savings. Quality improvement in healthcare is vital to ensure equality of access and efficient delivery of increasingly complex treatments. Every minor improvement is likely to have a significant cumulative gain for the system and, more specifically, for the patient. Marginal gains are, therefore, important. The shift towards minimally invasive surgery delivers not only short-term gains that have been well documented, but this meta-analysis demonstrates a more far reaching consequence of the approach. By potentially reducing the burden of SSIs for patients we can reduce suffering, the financial loss and life disruption in terms of need for carers and dependency on others. For the healthcare system, every avoidable return to hospital or primary care physician creates capacity for a system that can then function more efficiently for those in need. The financial savings are significant for both patient and healthcare system. This paper reiterates that the minimally invasive approach is one that is worth investing both in terms of proper quality assured training and in terms of capital expenditure on hardware. Perhaps a new solution to hardware procurement may help reduce costs further. The findings also raise questions about how these techniques can be reproduced on a global scale and especially in low and middle income countries. Although COVID-19 has temporarily halted widespread laparoscopy, recent guidance from Society of American Gastrointestinal and Endoscopic Surgeons (SAGES), the European Association of Endoscopic Surgeons (EAES) and the Association of Laparoscopic Surgeons of Great Britain and Ireland (ALGBI) have opened the way for laparoscopy to resume and there will inevitably by significant scrutiny of the key benefits [25-27]. We believe this paper forms a strong argument for a minimally invasive approach to colorectal procedures if undertaken in the correct manner with appropriate biohazard protection.

Robotic surgery for colorectal resections is a field in development. However, there is still no scientifically robust data that has shown any superiority of this technique to the laparoscopic approach in colorectal surgery [28]. The NBOCAP report has also shown that the number of centres and surgeons employing the robotic approach is steadily increasing. As it uses the same principles of minimally invasive approach as laparoscopic surgery, we feel that the benefits of lower SSI rates may also be seen in robotic surgery like laparoscopic surgery. This hypothesis has to be formally tested. 


\begin{tabular}{|c|c|c|c|c|c|c|c|c|}
\hline \multirow[b]{2}{*}{ Stuchy or Subgroup } & \multicolumn{2}{|c|}{ laparoscopic surgery } & \multicolumn{2}{|c|}{ open surgery } & \multirow{2}{*}{\multicolumn{2}{|c|}{ 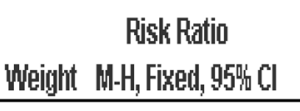 }} & \multirow{2}{*}{\multicolumn{2}{|c|}{$\begin{array}{c}\text { Risk Ratio } \\
\text { M-H, Fixed, 95唝 Cl }\end{array}$}} \\
\hline & Events & Total & Events & Total & & & & \\
\hline ALCASS TRIAL 2000 & 24 & 294 & 42 & 298 & $14.9 \%$ & $0.58[0.36,0.93]$ & $\rightarrow$ & \\
\hline Braga 2002 & 12 & 136 & 27 & 133 & $9.8 \%$ & $0.43[0.23,0.82]$ & $\rightarrow$ & \\
\hline COLOR 12005 & 20 & 535 & 16 & 545 & $5.7 \%$ & $1.27[0.67,2.43]$ & & \\
\hline COLOR $\| 2013$ & 79 & 697 & 30 & 345 & $18.6 \%$ & $1.00[0.70,1.44]$ & & $\rightarrow$ \\
\hline Curet 2004 & 2 & 25 & 1 & 18 & $0.4 \%$ & $1.44[0.14,14.60]$ & & \\
\hline Hasegawa et al 2003 & 1 & 24 & 3 & 26 & $1.0 \%$ & $0.36[0.04,3.24]$ & & \\
\hline Lacy et al 2002 & 8 & 111 & 19 & 108 & $6.9 \%$ & $0.41[0.19,0.90]$ & 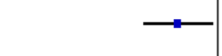 & \\
\hline LAFATRIALL 2011 & 14 & 209 & 26 & 191 & $9.7 \%$ & $0.49[0.26,0.91]$ & & \\
\hline LAFKONN TRIALL 2009 & 23 & 222 & 27 & 250 & $9.1 \%$ & $0.96[0.57,1.62]$ & & \\
\hline Leung et al & 10 & 167 & 17 & 170 & $6.0 \%$ & $0.60[0.28,1.27]$ & & \\
\hline MAARTENSE CROHNS 2006 & 0 & 30 & 8 & 30 & $30 \%$ & $0.06[0.00,0.98]$ & & \\
\hline MARRTENSE UC 2004 & 1 & 30 & 1 & 30 & $0.4 \%$ & $1.00[0.07,15.26]$ & & \\
\hline Milsom 2001 & 2 & 31 & 3 & 29 & $1.1 \%$ & $0.62[0.11,3.47]$ & & \\
\hline MRC CLAS8IC 2005 & 47 & 526 & 22 & 268 & $10.4 \%$ & $1.09[0.67,1.77]$ & & \\
\hline TANG ET AL 2001 & 5 & 118 & 4 & 118 & $1.4 \%$ & $1.25[0.34,4.54]$ & & \\
\hline Minslow et al 2002 & 5 & 37 & 5 & 46 & $1.6 \%$ & $1.24[0.39,3.97]$ & 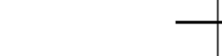 & - \\
\hline Total (95. Cl) & & 3192 & & 2605 & $100.0 \%$ & $0.76[0.64,0.90]$ & & 4 \\
\hline Total events & 253 & & 260 & & & & & \\
\hline $\begin{array}{l}\text { Heterogeneity } \mathrm{Ch}^{2}=21.74 \text {, df } \\
\text { Test for owerall effect: } \mathrm{Z}=3.18\end{array}$ & $\begin{array}{l}=15(P=0.11) \\
P=0.001)\end{array}$ & & & & & & $\begin{array}{|cc|}0.01 & 0.1 \\
\text { anours } & 1 \text { laparoscopic] }\end{array}$ & $\begin{array}{lll}1 & 10 & 100 \\
8 \text { Favours [open] }\end{array}$ \\
\hline
\end{tabular}

Fig. 6 Forest plot for surgical site infection combined with wound infection and abscess for laparoscopic versus open colorectal surgery

\section{Conclusions}

Our study has shown that modern laparoscopic surgery, in an era of more widespread adoption of the technique leads to a statistically significant lower incidence of SSIs compared to the open approach.

\section{Compliance with ethical standards}

Conflict of interest The authors declare that they have no conflict of interest.

Ethical approval This article does not contain any studies with human participants or animals performed by any of the authors.

Informed consent In this article no patient care was involved.

\section{References}

1. Public Health England (1991) Surveillance of surgical site infections in NHS hospitals in England. Public Health England, London, p 14
2. Jacobs V (1991) Goldstein, Minimally invasive colon resection (laparoscopic colectomy). Surg Laprosc Endosc 1(3):144-150

3. Law MSD, Pollock N, Letts L, Bosch J, Westmorland M (1998) Guidelines for Critical Review Form-Qualitative Studies. McMaster University Occupational Therapy Evidence-Based Practice Research Group

4. Hewett PJ et al (2008) Short-term outcomes of the Australasian randomized clinical study comparing laparoscopic and conventional open surgical treatments for colon cancer: the ALCCaS trial. Ann Surg 248(5):728-738

5. Veldkamp R et al (2005) Laparoscopic surgery versus open surgery for colon cancer: short-term outcomes of a randomised trial. Lancet Oncol 6(7):477-484

6. van der Pas MH et al (2013) Laparoscopic versus open surgery for rectal cancer (COLOR II): short-term outcomes of a randomised, phase 3 trial. Lancet Oncol 14(3):210-218

7. Vlug MS et al (2011) Laparoscopy in combination with fast track multimodal management is the best perioperative strategy in patients undergoing colonic surgery: a randomized clinical trial (LAFA-study). Ann Surg 254(6):868-875

8. Neudecker J et al (2009) Short-term outcomes from a prospective randomized trial comparing laparoscopic and open surgery for colorectal cancer. Br J Surg 96(12):1458-1467

9. Maartense S et al (2004) Hand-assisted laparoscopic versus open restorative proctocolectomy with ileal pouch anal anastomosis: a randomized trial. Ann Surg 240(6):984-991 (discussion 991-2) 
10. Maartense S et al (2006) Laparoscopic-assisted versus open ileocolic resection for Crohn's disease: a randomized trial. Ann Surg 243(2): 143-149 (discussion 150-3)

11. Jayne DG et al (2007) Randomized trial of laparoscopic-assisted resection of colorectal carcinoma: 3-year results of the UK MRC CLASICC Trial Group. J Clin Oncol 25(21):3061-3068

12. Braga $\mathrm{M}$ et al (2002) Laparoscopic versus open colorectal surgery: a randomized trial on short-term outcome. Ann Surg 236(6):759766 (discussion 767)

13. Curet MJ et al (2000) Laparoscopically assisted colon resection for colon carcinoma: perioperative results and long-term outcome. Surg Endosc 14(11):1062-1066

14. Hasegawa $\mathrm{H}$ et al (2003) Randomized controlled trial of laparoscopic versus open colectomy for advanced colorectal cancer. Surg Endosc 17(4):636-640

15. Lacy AM et al (2002) Laparoscopy-assisted colectomy versus open colectomy for treatment of non-metastatic colon cancer: a randomised trial. Lancet 359(9325):2224-2229

16. Leung KL et al (2004) Laparoscopic resection of rectosigmoid carcinoma: prospective randomised trial. Lancet 363(9416):1187-1192

17. Milsom JW et al (2001) Prospective, randomized trial comparing laparoscopic vs. conventional surgery for refractory ileocolic Crohn's disease. Dis Colon Rectum 44(1):1-8 (discussion 8-9)

18. Tang CL et al (2001) Randomized clinical trial of the effect of open versus laparoscopically assisted colectomy on systemic immunity in patients with colorectal cancer. Br J Surg 88(6):801-807

19. Winslow ER et al (2002) Wound complications of laparoscopic vs open colectomy. Surg Endosc 16(10):1420-1425

20. N.I.f.h.a.c.e.N (2019) Surgical site infections: prevention and treatment
21. Schwenk W et al (2005) Short term benefits for laparoscopic colorectal resection. Cochrane Database Syst Rev 3:Cd003145

22. Noel JK et al (2007) Minimally invasive colorectal resection outcomes: short-term comparison with open procedures. J Am Coll Surg 204(2):291-307

23. Yamamoto $\mathrm{S}$ et al (2008) Wound infection after a laparoscopic resection for colorectal cancer. Surg Today 38(7):618-622

24. RCS., NHS Digital, HQIP, National bowel cancer audit project 2019. 2019 https://www.nboca.org.uk/reports/annual-report-2019/ (30/05/2020)ACPGBI. Accessed 30 May 2020

25. ALSGBI, Laparoscopy in the COVID-19 environment. ALSGBI position statement. 2020, ALSGBI.org https://www.alsgb i.org/2020/04/22/laparoscopy-in-the-covid-19-environment-alsgb i-position-statement/. Accessed 30 May 2020

26. SAGES, Recommendations-surgical response-COVID19 (2020) SAGES website https://www.sages.org/recommendations-surgi cal-response-covid-19/. Accessed 30 May 2020

27. Morris SN et al (2020) Understanding the "Scope" of the problem: why laparoscopy is considered safe during the COVID-19 pandemic. J Minim Invasive Gynecol 27(4): 789-791

28. Jayne D et al (2017) Effect of robotic-assisted vs conventional laparoscopic surgery on risk of conversion to open laparotomy among patients undergoing resection for rectal cancer: The ROLARR randomized clinical trial. JAMA 318(16):1569-1580

Publisher's Note Springer Nature remains neutral with regard to jurisdictional claims in published maps and institutional affiliations. 